\title{
Operando State Diagnosis of Supported Ionic Liquid Phase Gas Purification Processes by a Resonant Perturbation Method
}

\author{
Vladimir Malashchuk, ${ }^{1,2}$, Andreas Jess ${ }^{1}$, Ralf Moos ${ }^{2}$ \\ 1 Department of Chemical Engineering, University of Bayreuth, 95440 Bayreuth, \\ 2 Department of Functional Materials, University of Bayreuth, 95440 Bayreuth, \\ Vladimir.malashchuk@uni-bayreuth.de
}

\begin{abstract}
Summary:
The ionic liquid (IL) 1-Ethyl-3-methylimidazolium methanesulfonate [EMIM][MeSO 3 impregnated on silica 90 packed to fixed-bed was analyzed by the resonant perturbation method. Therefore, the sample was positioned within a glass reactor in a microwave cavity to analyze resonance frequency and the inverse quality factor under sorption conditions. Two humidity sensors located before and after the fixed bed and allowed to calculate the amount of stored water in the IL. This allows for operando observation of changes of the resonant frequency and the inverse quality factor with the amount of water in the sample. The results showed a decrease in the resonant frequency and an increase in the inverse quality factor with increased water-sorption in the IL. Both parameters thus allow to derive the average water load on the system.
\end{abstract}

Keywords: Resonant-perturbation method, Water loading, Supported ionic liquid phase, Operando diagnosis

\section{Motivation}

The removal of water vapor from gas streams is a pretreatment process in the chemical industry to prevent catalyst poisoning, equipment damage caused by corrosion and the build-up of hydrates in natural gas pipelines [1]. For economical usage and efficiency, adsorption plants are used to reduce the water content in feed gases to very low dew points [2]. The commonly used setup contains fix-bed reactors filled with adsorbent materials such as silica gels, activated alumina, or molecular sieves [3]. Thereby, the water vapor flows through the fixed-bed until a breakthrough downstream of the fixed-bed is detected (by a humidity sensor) or a threshold value is reached. Then, a regeneration is initiated. Mathematical models based on the data from the humidity sensors up- and downstream of the fixed-bed predict the breakthrough time and state of the fixed-bed with respect to the load, the height of the mass transfer zone and the height of the unused bed.

In a recent approach, supported ionic liquid phase (SILP) materials are used as adsorbent agents in fixed bed reactor units. In a previous investigation, [EMIM][MeSO 3 supported on silica 90 was tested as adsorbent material for water, and the experimental data have shown their ability for storing water in the ionic liquid phase of the SILP material. This makes these systems interesting for technical application [4]. As the SILP material stores water, the ionic liquid phase changes gradually due to the increasing water content.

The analysis of the SILP material in a microwave resonator allows for observing operando changes in material properties during a sorption experiment. By analyzing scattering parameters while water vapor flows through the fixed bed, changes in the resonant frequency and quality factor are detected due to changes in the electrical material parameters of the bed.

\section{Experimental Method}

As a representative for an industrial fixed-bed adsorber, immobilized [EMIM][$\left[\mathrm{MeSO}_{3}\right]$ on silica 90 with a pore filling degree of $35 \%$ is placed in a quartz glass reactor in the center of a cavity resonator. The height of the fixed-bed corresponds to the cavity-height to minimize polarization effects. The resonator is connected to a network analyzer via two coaxial lines. Two loop coupling elements are used to excite the electromagnetic field into the cavity. Both resonant frequency and quality factor are calculated from the spectrum of the $S_{21}$ scattering parameters. At the resonant frequency, the maximum 
electric field strength is in the center of the cavity and does not change along the height of the fixed-bed (TM-mode, TM010). Details of the setup can be found in [5]. Due to the sorbed water in the supported IL, the dielectric properties of the fixed-bed spatially change and influence both the resonant frequency and the inverse quality factor. Since the electric field completely penetrates the whole sample, no spatial resolved but averaged changes of the dielectric material properties can be measured. The changes in the resonant frequency and the inverse quality factor are caused by the much higher electrical conductivity of the pure ionic liquids [EMIM][MeSO$\left.{ }_{3}\right]$ compared with its support silica 90. At constant temperature, the electrical conductivity of the pure IL increases with the amount of stored water. During the experiment, the water content of the nitrogen water mixture was verified by two humidity sensors upstream and downstream of the fixed bed. To consider the pressure loss along the fixed-bed, two absolute pressure sensors were used before and after the fixed-bed. With the data from the pressure sensors, humidity sensors, and the measured flow rate, the stored water content in the sample was calculated.

\section{Results}

Fig. 1 shows the sorbed amount of water $L_{\mathrm{H} 2 \mathrm{O}}$ in $\mathrm{kg}_{\text {H2O }}$ per $\mathrm{kg}_{\text {silica }}$ over time (A), the change in the resonant frequency fres $(B)$, and the inverse quality factor $1000 / Q(C)$. Before the sorption starts at $0.5 \mathrm{~h}$, the sample was flushed with dry nitrogen. At $0.5 \mathrm{~h}$, the sorption was initiated and the nitrogen/water mixture with water a partial pressure of 20 mbar flows through the fixedbed. Apparently, the sorbed amount of water in the sample increases (see. Fig. $1(\mathrm{~A})$ ), the resonant frequency decreases (B), and the inverse quality factor increases (C). At $1.5 \mathrm{~h}$, the steady state is reached and no more water can be sorbed in the sample at the operating conditions. From that point in time, the sorbed amount of water, the resonant frequency as well as the inverse quality factor do not change anymore. At $3 \mathrm{~h}$, the drying process (regeneration of the IL) was initiated by flushing the fixedbed with dry nitrogen. This leads to decreasing amount of sorbed water in the sample (Fig. $1(A)$ ), increasing resonant frequency (B), and decreasing of the inverse quality factor (C). As the point, when the sample is fully dry, no water is stored in the sample and the resonant frequency and the inverse quality factor return to their original values before the sorption. The increase in the inverse quality factor is related to increase in losses. At that point, the origin of the electrical losses, i.e. dielectric or ohmic losses, cannot be identified and further investigations are required.
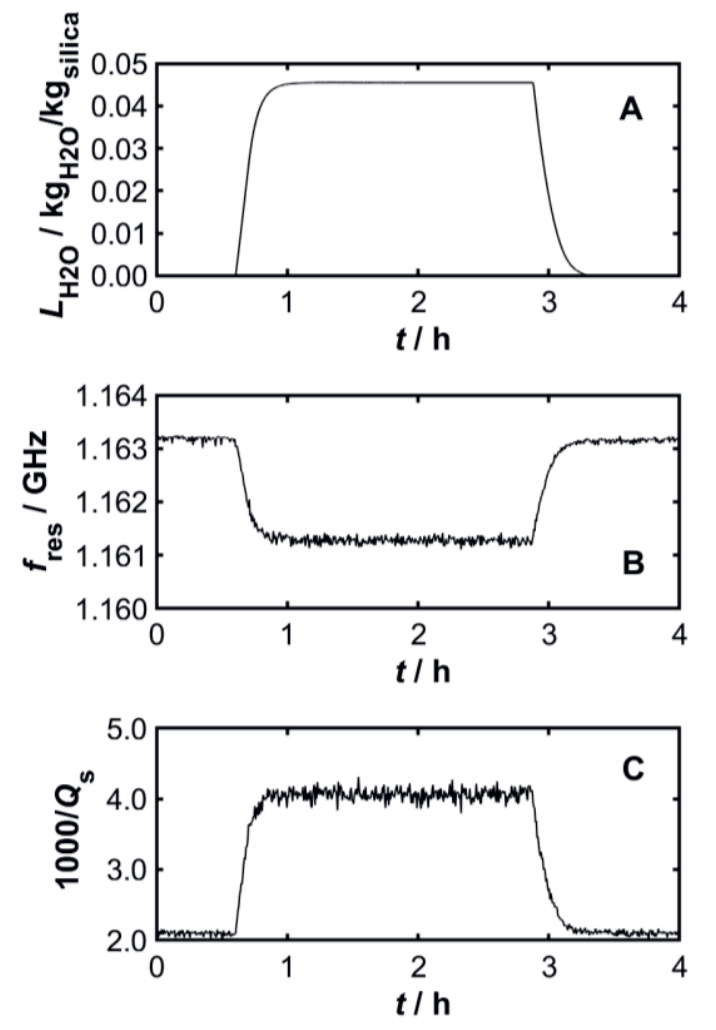

Fig. 1. Increase of sorbed water content $L_{\mathrm{H} 2 \mathrm{O}}$ correlates with decrease in resonance frequency $f_{\text {res }}$ and increase in inverse quality factor $1000 / \mathrm{Q} ; 50^{\circ} \mathrm{C}$, $P_{\mathrm{H} 2 \mathrm{O}}=20 \mathrm{mbar}, P_{\text {abs }}=1 \mathrm{bar}, m_{\text {sample }}=3.48 \mathrm{~g}$, total volume flow rate of $50 \mathrm{~L} / \mathrm{h}$ (STP), pore filling degree $\alpha=0.35$, mass loading of ionic liquid on the silica gel $\vartheta=0.38 \mathrm{~kg} ॥ / \mathrm{kg}_{\text {silica }}$

\section{Acknowledgements}

All authors are indebted to the German Research Foundation (DFG) for funding under grant (JE 257/21-2 and MO 1060/27-2)

\section{References}

[1] Arthur L. Kohl, Richard B. Nielsen: Gas Purification, Elsevier (1997), doi: 10.1016/B978-0-88415220-0.X5000-9

[2] A. Mersmann, B. Fill, R. Hartmann, S. Maurer: The Potential of Energy Saving by Gas-Phase Adsorption Processes, Chem. Eng. Technol., 23, 937-944 (2000)

[3] A. Jess, P. Wasserscheid: Chemical technology: An integral textbook, Wiley-VCH, Weinheim (2013), ISBN 978-3527304462

[4] F.R. Radakovitsch, A. Jess: Gas dehydration using the ionic liquid [EMIM][MeSO3] supported on silica gel - Structural and water vapor sorption properties, Chemical Engineering Journal, 398, 124689 (2020), doi: 10.1016/j.cej.2020.124689

[5] M. Dietrich, D. Rauch, A. Porch, R. Moos: A laboratory test setup for in situ measurements of the dielectric properties of catalyst powder samples under reaction conditions by microwave cavity perturbation: set up and initial tests, Sensors, 14, 16856-16868 (2014), doi: $10.3390 / \mathrm{s} 140916856$ 